Article

\title{
The Soluble Adenylyl Cyclase Inhibitor LRE1 Prevents Hepatic Ischemia/Reperfusion Damage Through Improvement of Mitochondrial Function
}

\author{
João S. Teodoro ${ }^{1,2, * \mathbb{C}}$, João A. Amorim $1,3,4 \oplus$, Ivo F. Machado ${ }^{1,2} \mathbb{D}$, Ana C. Castela ${ }^{1,2}$, \\ Clemens Steegborn ${ }^{5}$, David A. Sinclair ${ }^{4,6}$, Anabela P. Rolo ${ }^{1,2}$ and Carlos M. Palmeira ${ }^{1,2, *}$ \\ 1 Center for Neurosciences and Cell Biology of the University of Coimbra, 3004-504 Coimbra, Portugal; \\ Joao_Amorim@hms.harvard.edu (J.A.A.); ivofmachado@gmail.com (I.F.M.); \\ catycastela@hotmail.com (A.C.C.); anpiro@ci.uc.pt (A.P.R.) \\ 2 Department of Life Sciences of the University of Coimbra, 3000-456 Coimbra, Portugal \\ 3 IIIUC-Institute of Interdisciplinary Research of the University of Coimbra, 3030-789 Coimbra, Portugal \\ 4 Department of Genetics, Blavatnik Institute, Paul F. Glenn Center for the Biology of Aging, \\ Harvard Medical School, Boston, MA 02115, USA; david_sinclair@hms.harvard.edu \\ 5 Department of Biochemistry, University of Bayreuth, 95440 Bayreuth, Germany; \\ clemens.steegborn@uni-bayreuth.de \\ 6 Laboratory for Ageing Research, Department of Pharmacology, School of Medical Sciences, The University \\ of New South Wales, Sydney 2052, Australia \\ * $\quad$ Correspondence: jteodoro@ci.uc.pt (J.S.T.); palmeira@ci.uc.pt (C.M.P.); Tel.: +351-239-240-700 (J.S.T. \& C.M.P.)
}

Received: 13 May 2020; Accepted: 5 July 2020; Published: 11 July 2020

\begin{abstract}
Hepatic ischemia/reperfusion (I/R) injury is a leading cause of organ dysfunction and failure in numerous pathological and surgical settings. At the core of this issue lies mitochondrial dysfunction. Hence, strategies that prime mitochondria towards damage resilience might prove applicable in a clinical setting. A promising approach has been to induce a mitohormetic response, removing less capable organelles, and replacing them with more competent ones, in preparation for an insult. Recently, a soluble form of adenylyl cyclase (sAC) has been shown to exist within mitochondria, the activation of which improved mitochondrial function. Here, we sought to understand if inhibiting mitochondrial SAC would elicit mitohormesis and protect the liver from I/R injury. Wistar male rats were pretreated with LRE1, a specific SAC inhibitor, prior to the induction of hepatic $\mathrm{I} / \mathrm{R}$ injury, after which mitochondria were collected and their metabolic function was assessed. We find LRE1 to be an effective inducer of a mitohormetic response based on all parameters tested, a phenomenon that appears to require the activity of the $\mathrm{NAD}^{+}$-dependent sirtuin deacylase (SirT3) and the subsequent deacetylation of mitochondrial proteins. We conclude that LRE1 pretreatment leads to a mitohormetic response that protects mitochondrial function during I/R injury.
\end{abstract}

Keywords: mitochondria; ischemia/reperfusion; liver; soluble adenylyl cyclase; LRE1; sirtuin 3

\section{Introduction}

Ischemia/reperfusion (I/R) injury is common but multifactorial event in many surgical situations and, in some cases, in pathological conditions. It occurs when blood flow is restricted to at least part of an organ and is then restored. $\mathrm{I} / \mathrm{R}$ is characterized by cellular injury, a major decrease in mitochondrial function, and a concomitant decrease in both energetic homeostasis and metabolic competency.

Hypoxic conditions that occur during ischemia cause a metabolic shift away from mitochondrial oxidative phosphorylation (OXPHOS) towards glycolysis. During reperfusion, cells are further stressed due to a burst in oxygen availability and the rebalancing of $\mathrm{pH}$, among other factors $[1,2]$, 
leading to a burst in oxidative stress caused by reactive oxygen and nitrogen species (ROS and RNS, respectively) [3-5]. Another key initial player is intracellular calcium overload [5] and, later on, tissue inflammation.

Central to I/R injury are mitochondria [6-8]. These organelles, which are responsible for over $90 \%$ of a cells' ATP requirements, are highly sensitive to perturbations caused by I/R [9-11]. As such, strategies that protect mitochondria from I/R injury might be able to prevent tissue damage and save countless lives [9]. A strategy that has shown great potential in several studies hinges on the concept of mitohormesis [12,13], whereby low levels of injury are actually beneficial because they promote the autophagic removal of susceptible and damaged organelles, which are eventually replenished by newer ones, more capable of dealing with the injury. However, higher doses of insult do not generate a protective response strong enough to deal with the insult, resulting in cellular injury, cell death, and organ dysfunction [14]. One way to achieve a mitohormetic response is to prime mitochondria to deal with an injury using pharmacological or surgical means.

Achieving a mitohormetic response requires the activation of mitodynamic processes, such as fusion/fission of mitochondria, removal of injured units (mitophagy), and repopulation of the cell with newly generated mitochondria (biogenesis). This requires close communication between nuclear and mitochondrial genomes, as well as a tight signaling cascade of events that indicate which units to remove and which to replicate. Furthermore, post-translational modification of mitochondrial proteins by reversible phosphorylation/deacetylation by cAMP-dependent or sirtuin-dependent pathways is also crucial (reviewed in [14]).

cAMP is a ubiquitous second messenger involved in mitochondrial dynamics, metabolic homeostasis, and oxidative stress $[15,16]$. Ten adenylyl cyclases (AC) have so far been identified, but of great relevance to mitochondrial function was the identification of a soluble form (sAC), present in the mitochondrial matrix, aside from the already known transmembrane version $[17,18]$. sAC promotes the activation of protein kinase A (PKA), which elevates OXPHOS rates and decreases oxidative stress. There are some unanswered questions about the existence and function of PKA within mitochondria, in particular, its role in potentially regulating mitochondrial cAMP [19]. Regardless, the effects of cAMP in mitochondria are indisputable, and the increased mitochondrial function caused by activation of SAC is well accepted.

One of the effects of cAMP production in mitochondria is the activation of SirT3, a member of the sirtuin family of $\mathrm{NAD}^{+}$-dependent lysine deacylases/deacetylases [20]. SirT3 is the only native mitochondrial member of this family of enzymes whose activity has been directly linked to elevated mitochondrial function [21,22]. Interestingly, SirT3 is a known inducer of mitohormesis [23,24] and is vital for ROS detoxification [23], raising the possibility that sAC's effects on mitochondrial function might work via SirT3.

Based on these findings, we postulated that by inhibiting SAC with a specific inhibitor (LRE1), a hormetic response would be induced that would, by activating SirT3, prime hepatic mitochondria to be more resilient to injury caused by a deleterious event such as I/R.

\section{Results}

\subsection{Hepatic Mitochondrial Bioenergetics}

Data from isolated liver mitochondria from all experimental groups are represented in this section. As can be seen in Figure 1A,C, mitochondrial resting (initial) and repolarization (post-ADP phosphorylation) potentials were significantly diminished by the $I / R$, as previously reported [25]. LRE1 pretreatment of $30 \mathrm{~min}$ with a $1.2 \mathrm{mg} / \mathrm{kg}$ dose was sufficient to prevent said decrease, as was the case with the lag phase time (Figure 1D), i.e., the time required to phosphorylate a set amount of $\mathrm{ADP}$ and return the potential to a steady value. However, there were no differences, for any groups, in the depolarization potential (Figure 1B), i.e., the maximal decrease in potential as caused by a fixed $(200 \mathrm{nmol})$ amount of ADP. 
A
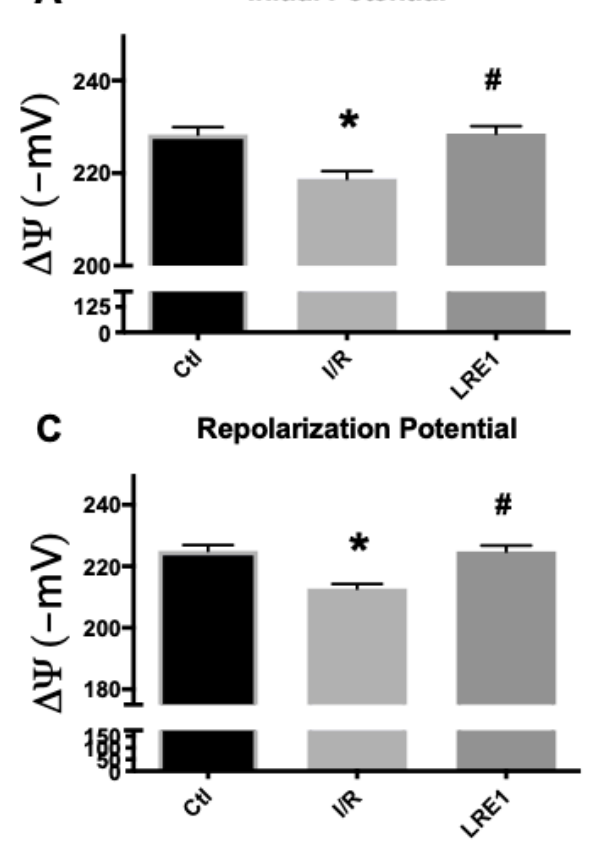

B
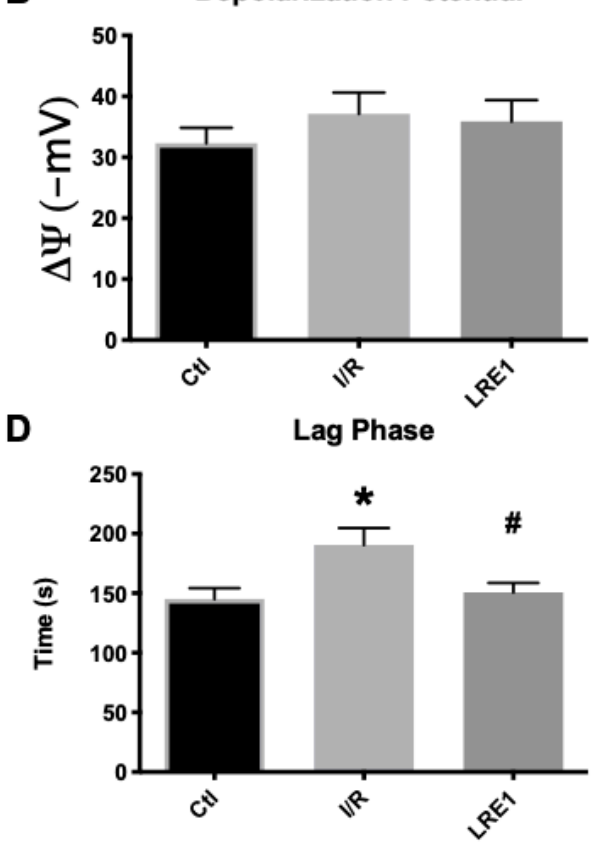

Figure 1. Mitochondrial membrane initial potential (A), ADP-driven depolarization potential (B), repolarization potential (C) and lag phase time (D) as evaluated by tetraphenylphosphonium ion $\left(\mathrm{TPP}^{+}\right)$ fluctuations and recorded with a $\mathrm{TPP}^{+}$-sensitive electrode. Data are means \pm SEM of 4 independent experiments. * indicates a statistically significant difference vs. sham operated controls (Ctl); \# indicates a statistically significant difference vs. ischemia/reperfusion (I/R).

Furthermore, simultaneous data on mitochondrial respiration, as evaluated with a Clark-type electrode, were recorded on the same samples as above. The obtained data are represented in Figure 2. In accordance with Figure 1, state 3 respiration (ADP-driven respiration) was affected by I/R injury, as was the $\mathrm{ADP} / \mathrm{O}$ ratio, i.e., the ratio indicating how much $\mathrm{ADP}$ can be phosphorylated by the same amount of oxygen consumed. Predictably, LRE1 pretreatment recovered both state 3 respiration and $\mathrm{ADP} / \mathrm{O}$ ratios (Figure 2A,D, respectively), but not state 4 respiration, i.e., post-ADP phosphorylation respiration (Figure $2 \mathrm{~B}$ ), a parameter that was also affected in the I/R group. Interestingly, the ICR ratio (respiratory control index) was not altered in any experimental group.

All these data from both Figures 1 and 2 indicate that LRE1 was a competent, albeit incomplete, effector of mitochondrial damage prevention through sAC inhibition. Further ex vivo testing of these mitochondrial preparations was performed. The data are represented in Figure 3 (mitochondrial swelling recording and ROS generation).

As can be seen in Figure 3A, mitochondrial resilience to $\mathrm{MPT}$ induction by means of a calcium challenge was significantly affected by $\mathrm{I} / \mathrm{R}$, a clear indicator of a reduction in available membrane potential (as seen in Figure 1) and overall poorer bioenergetic status. Once more, LRE1 was a competent preventer of I/R-driven alterations. Statistical analysis indicates that the I/R group was significantly lower than the other two groups at the 22 min mark. As for ROS generation, I/R drove ROS generation up in isolated liver mitochondria, an effect that was prevented by LRE1 pretreatment (Figure 3B).

Furthermore, we evaluated ATP levels within mitochondria subjected to I/R with and without LRE1 pretreatment. ATP quantification is represented in Figure 4. 

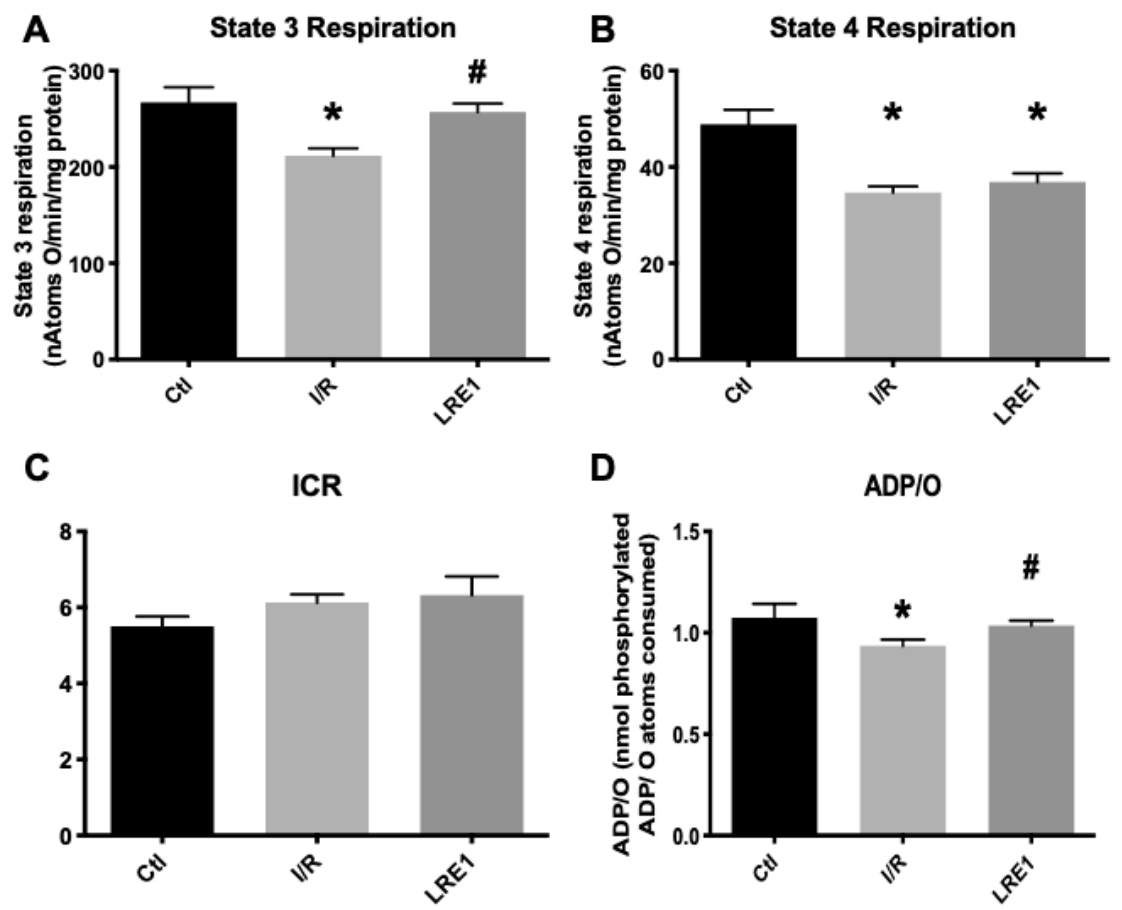

Figure 2. Mitochondrial oxygen consumption parameters (ADP-driven State 3 respiration, A; repolarization respiration State 4, B) and resulting ratios (Respiratory Control Index, ICR, C and; $\mathrm{ADP} / \mathrm{O}, \mathrm{D})$, as evaluated by $\mathrm{O}_{2}$ consumption and recorded with a Clark-type electrode. Data are means \pm SEM of 4 independent experiments. ${ }^{*}$ indicates a statistically significant difference vs. Ctl; \# indicates a statistically significant difference vs. I/R.
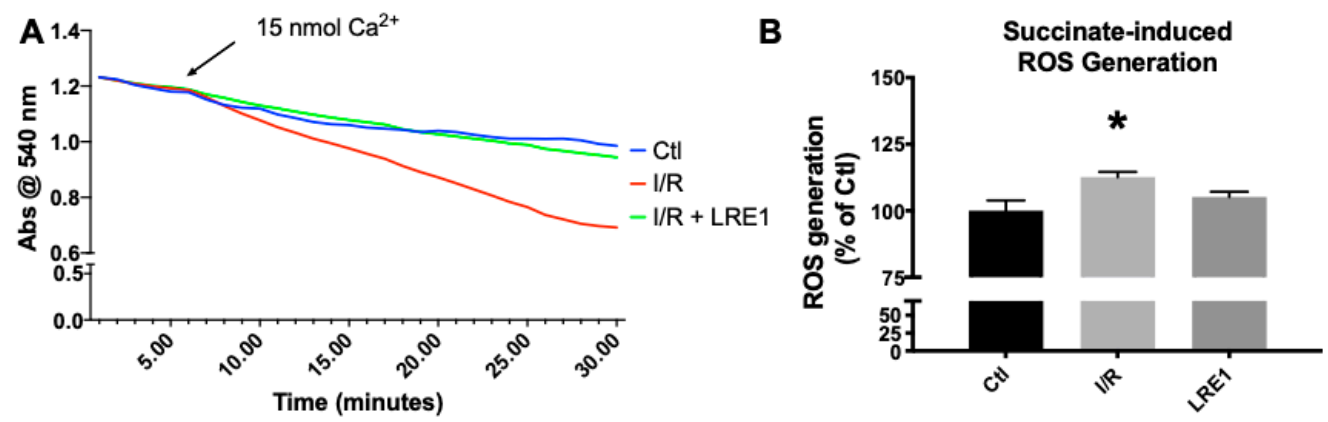

Figure 3. Mitochondrial swelling recording (A) and reactive oxygen species (ROS) generation (B). Swelling was recorded by following absorbance of light at $540 \mathrm{~nm}$ after a $\mathrm{Ca}^{2+}$ challenge, while ROS was evaluated by following $2^{\prime}, 7^{\prime}$-dichlorodihydrofluorescein diacetate (H2DCFDA) fluorescence for $10 \mathrm{~min}$. For panel A, lines indicate a representative recording of all of the assays, while panel B represents means \pm SEM of 4 independent experiments. * indicates a statistically significant difference vs. Ctl.

As expected, I/R caused a significant reduction in mitochondrial ATP content. However, LRE1 pretreatment tremendously elevated ATP levels when compared both with the $\mathrm{Ctl}$ and $\mathrm{I} / \mathrm{R}$ groups, a clear marker of the previously shown data, indicating that LRE1 pretreatment had ameliorated I/R deleterious events on hepatic mitochondrial function, even when compared with the Ctl group.

In conclusion, LRE1 pretreatment was capable of preventing most of the alterations to mitochondrial bioenergetics and evaluated functional parameters. 


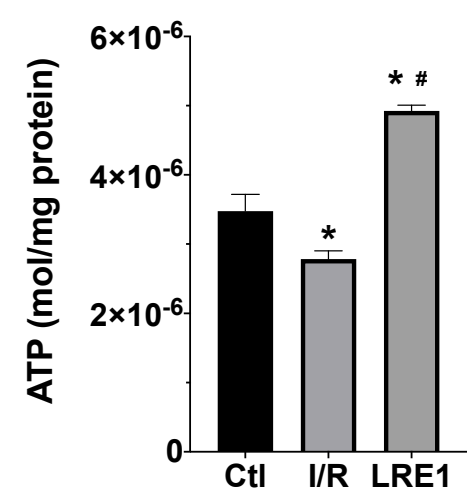

Figure 4. Liver mitochondria ATP content as evaluated with a bioluminescent assay. Data are means \pm SEM of 4 independent experiments. ${ }^{*}$ indicates a statistically significant difference vs. Ctl; \# indicates a statistically significant difference vs. I/R.

\subsection{Gene Expression and Protein Content}

To further evaluate the protective effects of sAC inhibition by LRE1 pretreatment on I/R injury, tissue samples and mitochondrial preparations were processed for gene expression and protein content analysis, respectively. Figures 5 and 6 illustrate the results obtained.
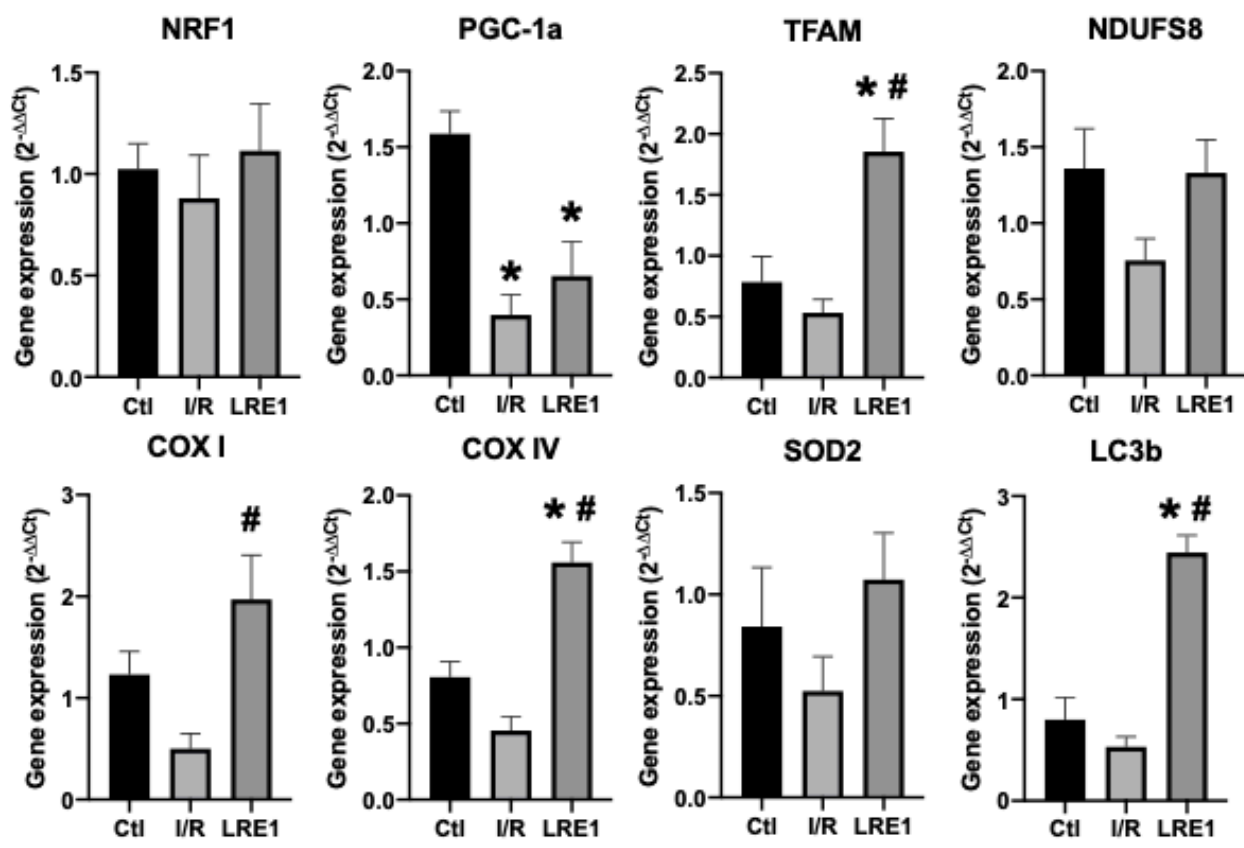

Figure 5. Liver gene expression evaluation by semi-qPCR. Data are means \pm SEM of 4 independent experiments. NRF1, nuclear respiratory factor 1; $P G C-1 a$, peroxisome proliferator-activated receptor gamma, coactivator 1 alpha; TFAM, mitochondrial transcription factor A; NDUFS8, NADH dehydrogenase iron-sulfur protein 8 , mitochondrial; $\operatorname{COX~I}$, cytochrome $\mathrm{c}$ oxidase subunit I; $C O X I V$, cytochrome c oxidase, subunit IV; SOD2, superoxide dismutase 2, mitochondrial; $L C 3 b$, microtubule-associated proteins $1 \mathrm{~A} / 1 \mathrm{~B}$ light chain $3 \mathrm{~B} .{ }^{*}$ indicates a statistically significant difference vs. $\mathrm{Ctl} ;$ \# indicates a statistically significant difference vs. I/R.

As can be seen in FLRE1 pretreatment significantly elevated the gene expression of the genes coding for mitochondrial complex IV (COX) subunits I and IV, the mitochondrial transcription factor A (TFAM), and microtubule-associated proteins 1A/1B light chain 3B $(L C 3 b)$, a regulator of mitophagy. Conversely, gene expression for the peroxisome proliferator-associated receptor gamma coactivator $1 \alpha$ 
(PGC-1a) expression was diminished by I/R injury and not rescued by LRE1 pretreatment. The elevation of TFAM and COX subunits expression indicates that the LRE1 pretreatment initiated mitochondrial biogenesis. The lack of effect on PGC-1 $\alpha$ and NRF1 genes expression might be due to a timing effect, since these players are involved in the early phases of mitochondrial biogenesis. Despite a clear trend towards the recovery of SOD2 levels, no statistical effect was observed at this time point. However, the elevation in $L C 3 b$ expression clearly indicates an increase in autophagic signaling that is known to be required for mitochondrial biogenesis and an improvement of mitochondrial function.
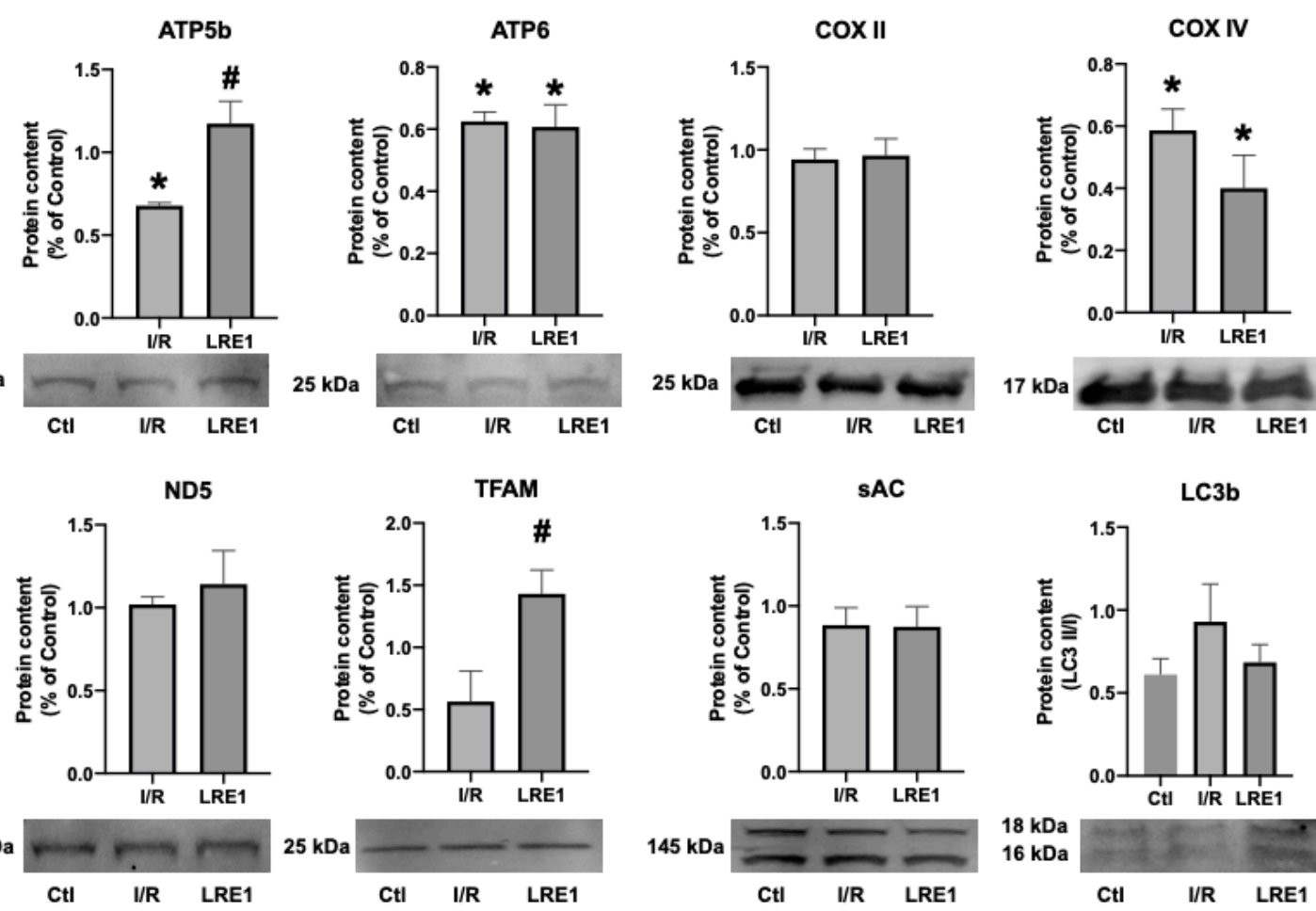

Figure 6. Hepatic mitochondrial protein content quantification by Western blot. Data are means \pm SEM of 4 independent experiments. ATP5b, ATP synthase F1 subunit beta, mitochondrial; ATP6, ATP synthase $\mathrm{F}_{\mathrm{O}}$ subunit 6, mitochondrial; COX II, cytochrome c oxidase subunit II; COX IV, cytochrome c oxidase, subunit IV; ND5, NADH-ubiquinone oxidoreductase chain 5; TFAM, mitochondrial transcription factor A; sAC, soluble adenylyl cyclase; LC3b, microtubule-associated proteins 1A/1B light chain 3B. * indicates a statistically significant difference vs. Ctl; \# indicates a statistically significant difference vs. I/R.

Next, we examined targets of sAC activity, namely, the commonly phosphorylated residues of mitochondrial proteins. cAMP generated by sAC within mitochondria has been shown to lead to the activation of PKA, thus contributing to the phosphorylation of several proteins within mitochondria [26,27]. Figure 7 shows the results obtained, where it is visible that LRE1 pretreatment led to the decrease in phosphorylation of total mitochondrial protein extracts.

A known target of cAMP is the mitochondrial matrix $\mathrm{NAD}^{+}$-dependent deacetylase sirtuin 3 (SirT3). SirT3 is a known positive effector of mitochondrial function, and thus insight into its levels and activity could help explain the effects of LRE1. In fact, SirT3 levels have been negatively correlated with elevated cAMP levels [26,27]. As such, we looked into the mitochondrial SirT3 content and mitochondrial acetylation levels. These data are presented in Figure 8.

The data from Figure 8 show that SirT3 levels trended downward in response to I/R, while LRE1 pretreatment was sufficient to prevent SirT3 content reduction and, concomitantly, led to the reduction in the presence of acetylated lysine residues in mitochondrial preparations. Since the presence of acetylated lysines in mitochondria is correlated with a decrease in mitochondrial OXPHOS capacity [26,27], it is likely that the protection of mitochondrial function provided by LRE1 pretreatment against I/R injury 
is linked to the activation of SirT3 and an increase mitochondrial function and ATP that prevents mitochondrial dysfunction and cell death in response to I/R injury.
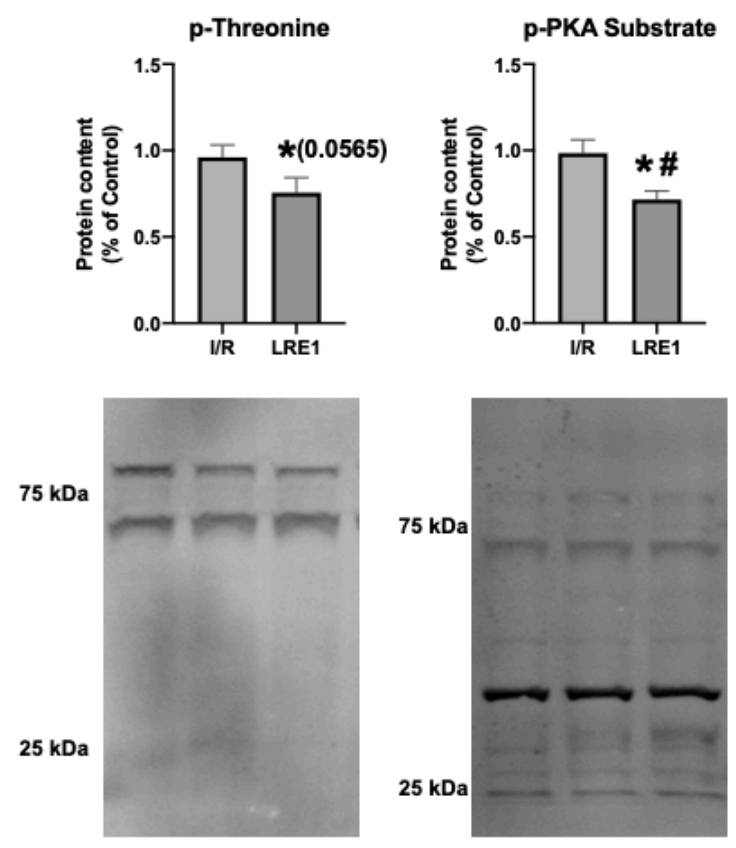

Figure 7. Hepatic mitochondrial protein phosphorylation content quantification by Western blot. Data are means \pm SEM of 4 independent experiments. p-threonine, phosphorylated threonine residues; p-PKA substrate, phosphorylated PKA substrate. * indicates a statistically significant difference vs. Ctl; \# indicates a statistically significant difference vs. I/R.
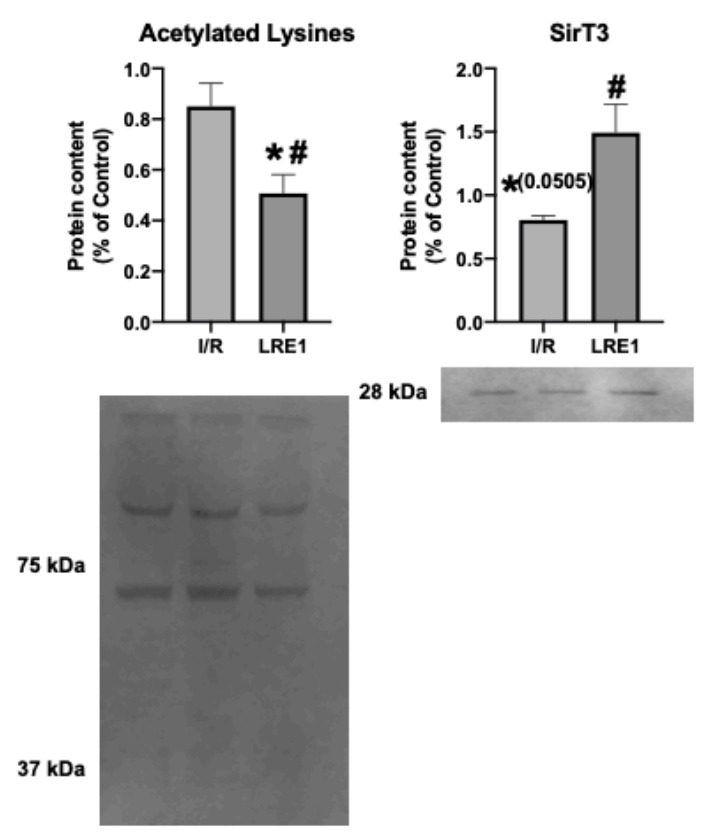

Figure 8. Hepatic mitochondrial protein acetylation status and $\mathrm{NAD}^{+}$-dependent deacetylase sirtuin 3 (SirT3) quantification by Western blot. Data are means \pm SEM of 4 independent experiments. SirT3, Sirtuin 3. * indicates a statistically significant difference vs. Ctl; \# indicates a statistically significant difference vs. I/R. 


\section{Discussion}

Ischemia/reperfusion (I/R) injury is a common phenomenon in various surgical settings, stroke, and chronic diseases such as cardiovascular and cerebrovascular diseases. The removal and restoration of blood flow is a potent inducer of cellular oxidative damage, organelle dysfunction, cellular death, and ultimately organ failure if enough widespread damage occurs [28]. Novel avenues of preventing this damage could be the difference between life and death. Given the central role of mitochondria in $\mathrm{I} / \mathrm{R}$, if these strategies are able to prevent mitochondrial dysfunction, then there is a real potential to these therapies. As such, exploring the mitohormetic capacity is a novel avenue to numerous diseases where mitochondrial function is involved [14,29].

A critical regulator of mitochondrial function is cAMP. This ubiquitous second messenger is produced by the adenylyl cyclase (AC) family of enzymes, of which there are numerous members. Of great interest to mitochondrial biology (given their existence in these organelles) are: (1) a transmembrane $\mathrm{AC}$, bound to the outer membrane which produces cAMP that does not permeate the mitochondrial membrane and thus does not affect OXPHOS and other matrix biochemical reactions [30] and (2) a soluble, free form (sAC) present in the matrix that produces virtually all of the cAMP having effects in mitochondria [31]. In response to carbon dioxide, sAC is activated and generates cAMP that ultimately leads to an increase in mitochondrial function, i.e., greater rates of OXPHOS function that ultimately lead to higher levels of the generated ATP $[15,32]$. Given this, our rationale was that if we can prime mitochondria through a mitohormetic effect to protect them against I/R injury, then the complications associated with this phenomenon could be better tolerated or downright prevented. This reasoning was already hinted at by a sAC knockout mouse model, in which, SAC removal dramatically elevated OXPHOS components [33].

In this study, we directly targeted mitochondrial sAC with a novel compound called LRE1 [34], which is known to allosterically inhibit sAC by binding to the same site as bicarbonate, thereby preventing its activation and reducing cAMP generation. In this and other studies, LRE1 treatment was accompanied by a reduction in mitochondrial OXPHOS respiratory chain complex IV cytochrome $c$ oxidase (COX) activity [34]. We hypothesized that pretreatment with LRE1 via its direct injection into the portal vein of animals about to experience surgery-induced $I / R$, would inhibit mitochondrial sAC and elevate mitochondrial capacity via mitohormesis.

Our data are consistent with this hypothesis. Most of the hepatic mitochondrial parameters we evaluated were reversed to sham-operated control values, despite an I/R event. Mitochondrial membrane potential $(\Delta \Psi)$ was also maintained, as well as the efficiency of the OXPHOS system, due to the prevention of lag phase elevation (Figure 1). Similarly, the maintenance of the ADP/O ratio and elevated state 3 respiration rates indicated a highly competent OXPHOS system post-I/R (Figure 2). ATP was also dramatically elevated, and as one would expect, mitochondrial function was improved.

Mitochondrial calcium tolerance capacity, as evaluated by swelling induction in response to free $\mathrm{Ca}^{2+}$ ions, was retained by LRE1 pretreatment, as well as prevention of oxidative stress as measured by $\mathrm{H}_{2} \mathrm{O}_{2}$ generation in isolated mitochondria (Figure 3). Because both $\mathrm{Ca}^{2+}$ mitochondrial metabolism and oxidative stress are hallmarks of $\mathrm{I} / \mathrm{R}$ injury and both have been involved in mitochondrial sAC-cAMP biology [32,33], it is reasonable to conclude that LRE1 pretreatment induced a mitohormetic response that elevated calcium tolerance and diminished ROS.

To further test our hypothesis, we next examined the expression of key mitochondrial biogenesis genes and genes coding for proteins of the respiratory chain and found that the TFAM genes, as well as genes encoding for cytochrome $c$ oxidase (COX) subunits I and IV, were elevated by LRE1 pretreatment when compared with the I/R group (Figure 4). Interestingly, the gene coding for the master regulator of mitochondrial biogenesis, PGC-1 $\alpha$, was not elevated by LRE1 pretreatment. Since PGC-1 $\alpha$ is the initial player in mitochondrial biogenesis [35], we attribute this to $P G C-1 \alpha$ gene activation occurring prior to the time point analyzed. The expression of the SOD2 gene showed a clear trend towards recovery, and given our ROS generation data (Figure 3), we strongly suspect that it is once more a time point issue. Finally, since the gene coding for $L C 3 b$, a key player in autophagic processes (which 
also includes mitophagy [36]) was significantly elevated by LRE1 pretreatment, we conclude that the cellular benefits during I/R injury were likely mediated by mitohormesis-induced mitophagy.

We also hypothesized that PKA activity was reduced by LRE1, with a concomitant reduction of cAMP generation. In Figure 6, we show that LRE1 pretreatment significantly downregulated PKA activation, as indicated by a reduction in p-threonine and p-Serine signals, two main targets of PKA $[37,38]$. Interestingly, despite claims in the literature that SirT3 is positively regulated by the sAC/cAMP/PKA axis [20,39], we found that SirT3 protein content is elevated by LRE1 pretreatment and is associated with a decrease in the acetylation levels of mitochondrial proteins. This finding helps to explain the improvement of mitochondrial function in LRE1-pretreated hepatic mitochondria post-I/R.

In conclusion, our data support a model in which inhibition of SAC by LRE1 induces a mitohormetic response that protects mitochondria from a large insult such as the one caused by I/R. Future work may include the testing of LRE1 in other injuries relevant to human health such as stroke, transplantation or acute kidney injury.

\section{Materials and Methods}

\subsection{Materials}

Except when specifically indicated, all reagents were purchased from Sigma-Aldrich (St. Louis, MO, USA) and were of the highest grade of purity commercially available.

LRE1 was dissolved in DMSO and originated from Nanosyn (Santa Clara, CA, USA), and its identification and specificity was reported in [40].

\subsection{Animals}

12 male Wistar rats (aged 12 weeks, Charles River Laboratories (Wilmington, MA, USA) were housed under standard conditions $(12 \mathrm{~h}$ light/dark cycles, with ambient temperature and humidity control) and had free access to rodent chow and acidified water. Animals were allowed to acclimatize for 1 week before experimental procedures. All studies were conducted in accordance with the principles and procedures outlined as "3Rs" in the guidelines of EU (1986/609/EEC and 2010/63/EU), FELASA and the National Centre for the 3Rs (the ARRIVE) and were approved by the Animal Care Committee of the Center for Neurosciences and Cell Biology of Coimbra. We also applied the principles of the ARRIVE guideline for data management and interpretation, and all efforts were made to minimize the number of animals used and their suffering, as standard [41]. This experimental project was approved by the CNC ORBEA (President: Dr. Catarina Oliveira), and was given the reference number ORBEA_150_2016_04112016 on 4 November 2016.

\subsection{Surgical Induction of Ischemia/Reperfusion}

Animals were randomly divided into 3 experimental groups: (a) sham operated controls, Ctl; (b) hepatic ischemia/reperfusion (I/R); and c) hepatic I/R but with a $30 \mathrm{~min}$ pretreatment with LRE1 $1.2 \mathrm{mg} / \mathrm{kg}$ injected in the hepatic artery (LRE1). LRE1 dosage was calculated from a previous work [34]. All animals were anesthetized with a cocktail of ketamine/chlorpromazine (both $50 \mathrm{mg} / \mathrm{kg}$ ).

A model of partial hepatic ischemia (70\%) was used to prevent mesenteric venous congestion by allowing decompression of portal flow through the right and caudate lobes, as previously reported [42]. After being subjected to a midline laparotomy, the hepatic artery and portal vein to the left and median liver lobes were clamped for $90 \mathrm{~min}$, after which a reperfusion phase (lasting $12 \mathrm{~h}$ ) was initiated by the removal of the clamps.

\subsection{Mitochondrial Isolation}

After the reperfusion period, animals were sacrificed by cervical dislocation and liver mitochondria were isolated using a standard isolation protocol [43], with modifications [44]. All procedures were conducted on ice or ice-cold equipment to prevent sample degradation. Briefly, livers were excised 
from the organisms and thinly minced with scissors in homogenization buffer ( $250 \mathrm{mM}$ sucrose, 0.5 mM EGTA, $10 \mathrm{mM}$ HEPES, 0.1\% fat-free bovine serum albumin, and BSA; pH 7.4). Medium was decanted and replaced twice to remove the maximal amount of blood possible. Tissue pieces were then homogenized with a Potter-Elvehjem tissue homogenizer. Liver homogenate was then decanted into centrifuge tubes and spun at $1000 \times \mathrm{g}$ for $10 \mathrm{~min}$ at $4{ }^{\circ} \mathrm{C}$. Supernatant was decanted into new tubes and was again spun for $10 \mathrm{~min}$ at $4{ }^{\circ} \mathrm{C}$, but at $10,000 \times g$. The supernatant was aspirated and the mitochondrial pellet was resuspended in fresh homogenization buffer, after which the previous centrifugation cycle was performed again. The supernatant was aspirated and the pellet was resuspended in washing buffer (250 mM sucrose and $10 \mathrm{mM}$ HEPES; pH 7.4). Samples were again immediately spun in the same conditions as mentioned above, and this last step was performed twice to obtain an ultrapure mitochondrial pellet. Mitochondrial protein content was quantified by the Biuret method calibrated with BSA [45].

\subsection{Mitochondrial Oxygen Consumption Evaluation}

Mitochondrial oxygen consumption was polarographically determined using a Clark-type electrode, as previously described [46]. Reactions were conducted at $25^{\circ} \mathrm{C}$ in a water bath-jacketed chamber with magnetic stirring. Mitochondria $(1 \mathrm{mg})$ were placed in $1.3 \mathrm{~mL}$ of standard mitochondrial respiratory buffer $\left(130 \mathrm{mM}\right.$ sucrose, $50 \mathrm{mM} \mathrm{KCl}, 5 \mathrm{mM} \mathrm{MgCl}_{2}, 5 \mathrm{mM} \mathrm{KH}_{2} \mathrm{PO}_{4}, 50 \mu \mathrm{M}$ EDTA, and $5 \mathrm{mM}$ HEPES; pH 7.4) supplemented with $2 \mu \mathrm{M}$ rotenone to prevent respiratory chain Complex I contribution and to prevent retrograde electronic flow. Mitochondria were energized with $5 \mathrm{mM}$ succinate, and state 3 respiration was initiated by the addition of $200 \mathrm{nmol}$ ADP. State 3, state 4, Respiratory Control Index, and ADP/O ratios were calculated according to a previous work $[43,47]$.

\subsection{Mitochondrial Membrane Potential ( $\Delta \Psi)$ Measurement}

$\Delta \Psi$ was estimated using an ion-selective electrode that evaluates the intercompartmental distribution of the tetraphenylphosphonium ion $\left(\mathrm{TPP}^{+}\right)$, a highly soluble ion that freely traverses the inner mitochondrial membrane in direct accordance to $\Delta \Psi$, as previously reported [48]. Voltage response of the $\mathrm{TPP}^{+}$-selective electrode to the logarithmic $\mathrm{TPP}^{+}$concentration was linear with a slope of $59 \pm 1$, in accordance with the Nernst equation. These assays were simultaneously performed with the oxygen consumption ones, as described above. A matrix volume of $1.1 \mu \mathrm{L} / \mathrm{mg}$ protein was assumed.

\subsection{Mitochondrial Permeability Transition Recording}

Mitochondrial swelling was evaluated by changes in light scattering as spectrophotometrically measured at $540 \mathrm{~nm}$, in a water bath-jacketed Helios Alpha Spectrophotometer (Thermo Electron Corporation, Waltham, MA, USA) [49]. Reactions were performed at $25^{\circ} \mathrm{C}$, and recording was started by the addition of $1 \mathrm{mg}$ of mitochondrial preparation to $2 \mathrm{~mL}$ of swelling medium ( $200 \mathrm{mM}$ sucrose, $10 \mathrm{mM}$ HEPES, $1 \mathrm{mM} \mathrm{KH}_{2} \mathrm{PO}_{4}$, and $10 \mu \mathrm{M}$ EGTA; pH 7.4) supplemented with $2 \mu \mathrm{M}$ rotenone and $5 \mathrm{mM}$ succinate. A negative control reaction was also supplemented with $1 \mu \mathrm{M}$ cyclosporin $\mathrm{A}$, a known mitochondrial permeability transition inhibitor. After $5 \mathrm{~min}$ of basal absorbance recording, $15 \mathrm{nmol}$ $\mathrm{Ca}^{2+}$ was added to all reactions and absorbance recording was performed for an additional $25 \mathrm{~min}$.

\subsection{Mitochondrial Reactive Oxygen Species (ROS) Generation Quantification}

ROS generation was fluorometrically determined using a Victor3 plate reader (Perkin-Elmer, Waltham, MA, USA), by recording the fluorescence emitted by $2^{\prime}, 7^{\prime}$-dichlorodihydrofluorescein diacetate $\left(\mathrm{H}_{2} \mathrm{DCFDA}\right)$ at $538 \mathrm{~nm}$ after excitation at $485 \mathrm{~nm}$ [50]. In brief, $1 \mathrm{mg}$ of isolated mitochondria were incubated in standard respiratory medium (as above) for $30 \mathrm{~min}$, with $5 \mathrm{mM}$ succinate and $50 \mu \mathrm{M}$ $\mathrm{H}_{2}$ DCFDA (in DMSO), at $37^{\circ} \mathrm{C}$. After 3 min centrifugation at $10,000 \times g$ to pellet mitochondria, medium was refreshed to remove excess fluorescent probe, after which, $200 \mu \mathrm{L}$ medium (corresponding to $0.2 \mathrm{mg}$ ) was loaded into 96-well plate wells. Fluorescence was recorded for $15 \mathrm{~min}$ to calculate a rate of ROS generation. Data are expressed as arbitrary relative fluorescence units (RFUs). 


\subsection{Mitochondrial ATP Content Evaluation}

ATP levels were quantified by use of an ATP Kit (Sigma-Aldrich), according to manufacturer's instructions. Briefly, ATP was extracted by adding $25 \mu \mathrm{L} 2.5 \mathrm{M} \mathrm{KOH}$ to $1 \mathrm{mg}$ of mitochondria. Samples were vortexed for a few seconds and centrifuged at $4{ }^{\circ} \mathrm{C}$ and $18,000 \times g$ for $10 \mathrm{~min}$. Supernatants were collected and 100-150 $\mu \mathrm{L}$ of $1 \mathrm{M} \mathrm{K}_{2} \mathrm{HPO}_{4}$ was added, in an amount sufficient for pH neutralization. Samples were then immediately placed on liquid $\mathrm{N}_{2}$ and stored at $-80{ }^{\circ} \mathrm{C}$ for later use. On assay day, ATP was quantified with the bioluminescent kit in a Victor3 plate reader.

\subsection{0. $q P C R$}

RNA extraction followed a standard protocol [51]. Briefly, liquid $\mathrm{N}_{2}$ flash-frozen mitochondria were thawed in guanidinium thiocyanate (Tri Reagent, Sigma-Aldrich) and mixed with 0.2 volumes of pure chloroform. After a 10 min centrifugation at $4{ }^{\circ} \mathrm{C}$ and $12,000 \times g$, the upper translucid phase containing RNA was pipetted to a RNAase-free tube, and a clean-up protocol involving $70 \%$ ethanol was performed. RNA was desiccated at room temperature and resuspended in molecular grade ultrapure water (Sigma-Aldrich). RNA was quantified using a NanoDrop One instrument (Thermo-Fisher, Waltham, MA, USA) and cDNA was synthesized using the iScript cDNA Synthesis Kit (Bio-Rad, Hercules, CA, USA). cDNA samples were then analyzed by qPCR (CFX 96 Touch, Bio-Rad) using the SsoAdvanced Universal SYBR Green Supermix Kit (Bio-Rad). Gene expression quantification was performed using the $2^{-\Delta \Delta C t}$ method [15]. Primers used are listed in Table 1 . They were previously tested in-house to verify that reaction efficiency was of at least $90 \%$.

Table 1. qPCR primers used.

\begin{tabular}{|c|c|c|}
\hline Primer Name & Sequence & NCBI Nucleotide's Accession Number \\
\hline COX I Forward & CCA GTA TTA GCA GCA GGT ATC & \multirow{2}{*}{ KY_754542.1 } \\
\hline COX I Reverse & CCG AAG AAT CAG AAT AGG TGT T & \\
\hline COX IV Forward & GGC AGA ATG TTG GCT ACC & \multirow{2}{*}{ NM_017202.1 } \\
\hline COX IV Reverse & GCA TAG TCT TCA CTC TTC ACA A & \\
\hline$L C 3 b$ Forward & CTT CAG GTG TGC AAT GCT GG & \multirow{2}{*}{ NM_022867.2 } \\
\hline LC3b Reverse & TGG CTC TCT TCC TGT TGC TG & \\
\hline NDUFS8 Forward & AGT GTA TCT ACT GTG GTT & \multirow{2}{*}{ NM_001106322.2 } \\
\hline NDUFS8 Reverse & TAG CTT CTC CTT GTT GTA & \\
\hline NRF1 Forward & GGA TTC ATT ATG GCG GAA GTA A & \multirow{2}{*}{ NM_001100708.1 } \\
\hline NRF1 Reverse & AGT TGC TGT GGC GAG TTA & \\
\hline PGC-1 $\alpha$ Forward & CTG CTC TTG AGA ATG GAT ATA CTT & \multirow{2}{*}{ NM_031347.1 } \\
\hline PGC-1 $\alpha$ Reverse & CATACT TGC TCT TGG TGG AA & \\
\hline SOD2 Forward & CAC TGT GGC TGA GCT GTT GT & \multirow{2}{*}{ NM_017051.2 } \\
\hline SOD2 Reverse & TCC AAG CAA TTC AAG CCT CT & \\
\hline TFAM Forward & AAA TGG CTG AAG TTG GGC GAA GTG & \multirow{2}{*}{ BC062022.1 } \\
\hline TFAM Reverse & AGC TTC TTG TGC CCA ATC CCA ATG & \\
\hline $18 S$ Forward & GTA ACC CGT TGA ACC CCA TT & \multirow{2}{*}{ NR_046239.1 } \\
\hline 18S Reverse & CCA TCC AAT CGG TAG TAG CG & \\
\hline
\end{tabular}

\subsection{Western Blotting}

Precisely, $1 \mathrm{mg}$ of flash-frozen mitochondria was lysed in ice-cold RIPA buffer supplemented with a cocktail of protease (Sigma-Aldrich) and phosphatase inhibitors (Pierce, Waltham, MA, USA) and diluted 1:1 in Laemmli buffer with 5\% $\beta$-mercaptoethanol. Precisely, $50 \mu \mathrm{g}$ of mitochondrial/Laemmli 
mix was loaded into homemade SDS-polyacrylamide gels or homemade TGX gels (both from Bio-Rad). Gel percentages depended on target protein size, but roughly targets that weighted over $80 \mathrm{kDa}$ were blotted in $8 \%$ gels, while targets below $30 \mathrm{kDa}$ were blotted in $15 \%$ gels; intermediate weights were blotted in 10\% acrylamide gels. Samples were then electrophoresed at constant voltage and transferred into PVDF membranes by means of a Trans-Blot Turbo apparatus (Bio-Rad). In the case of the TGX gels, these were imaged before transfer in a Bio-Rad's Gel Doc EZ Imager. After transfer, membranes were blocked with blocking buffer (Thermo-Fisher) for $2 \mathrm{~h}$ at room temperature before being cut and exposed overnight at $4{ }^{\circ} \mathrm{C}$ to primary antibodies (1:1000), dissolved in TBS-T with $5 \%$ blocking solution (listed in Table 2). The following day, membranes were washed 3 times for 20 min each time with TBS-T before incubation for $1 \mathrm{~h}$ with secondary antibodies (1:2500). Membranes were again washed 3 times for $20 \mathrm{~min}$ each time and incubated for 5-30 min with Qdot 625 streptavidin conjugate substrate (Thermo Fisher) in light-protected tubes (1:2500). Finally, membranes were imaged in the same Gel Doc EZ system as above. Blots were quantified using Bio-Rad's Image Lab software version 6.0.1.

Table 2. List of antibodies used for Western blotting.

\begin{tabular}{cccc}
\hline Antibody Name & Source Host & Supplier & Reference Number\# \\
\hline Acetylated lysines & Rabbit & Cell Signaling Technologies & 9441 \\
\hline ADCY10/sAC & Rabbit & Abcam & Ab82854 \\
\hline ATP5B & Rabbit & Aviva Systems Biology & ARP48185 \\
\hline ATP6 & Rabbit & LifeSpan BioSciences & LS-C352532 \\
\hline COX II & Rabbit & LifeSpan BioSciences & LS-C330986 \\
\hline COX IV & Rabbit & Thermo-Fisher & PA5-19471 \\
\hline ND5 & Rabbit & LifeSpan BioSciences & LS-C368770 \\
\hline LC3B & Rabbit & Sigma-Aldrich & L7543 \\
\hline p-PKA substrates & Rabbit & Cell Signaling Technologies & 9624 \\
\hline SIRT3 & Rabbit & Cell Signaling Technologies & 2627 \\
\hline TFAM & Rabbit & Aviva Systems Biology & ARP36993 \\
\hline p-threonine & Mouse & Qiagen & W10132 \\
\hline Biotin-XX, antimouse IgG & Goat & Thermo Fisher & W10132 \\
\hline Biotin-XX, antirabbit IgG & Goat & Thermo Fisher & W10142 \\
\hline
\end{tabular}

\subsection{Statistical Analysis}

Represented data are typically means \pm SEM of a $n$ of 4 different animals per group. Statistical significance was determined using one- or two-way ANOVA tests with a Tukey or Holm-Sidak, post hoc correction ran on GraphPad Prism 8 software for Mac OS (GraphPad, La Jolla, CA, USA). A $p$ value $\leq 0.05$ was considered statistically significant.

Author Contributions: J.S.T., A.P.R., C.S., D.A.S., and C.M.P. designed the study; J.S.T., J.A.A., A.P.R., I.F.M., A.C.C., and C.M.P. performed the assays; C.S. provided LRE1.; J.S.T., A.P.R., C.M.P., and D.A.S. interpreted data and wrote and/or edited the manuscript. All authors have read and agreed to the published version of the manuscript.

Funding: This work was financed by the European Regional Development Fund (ERDF), through the Centro 2020 Regional Operational Programme: project CENTRO-01-0145-FEDER-000012-HealthyAging2020, the Portugal 2020-Operational Programme for Competitiveness and Internationalisation, and the Portuguese national funds via FCT-Fundação para a Ciência e a Tecnologia, I.P.: project POCI-01-0145-FEDER-016770, as well as by UID/NEU/04539/2013 (CNC.IBILI Consortium strategic project). The work performed by the Sinclair laboratory was funded by an R01 grant from the National Institutes of Health (NIH) and the National Institute of Diabetes and Digestive and Kidney Diseases (NIDDK) (R01 DK100263). J.S.T. was a recipient of an FCT postdoctoral grant and is now a recipient of a CEEC researcher grant from FCT and CNC (SFRH/BPD/94036/2013 and CEECIND/4400/2017, respectively). I.F.M. and A.C.C. are recipients of a research grant (HEALTHYAGING 2020 
CENTRO-01-0145-FEDER-000012). We further acknowledge support by DFG (grant STE 1701/11 to CS) and by Pfleger-Stiftung (mitoSirtuins to C.S.).

Acknowledgments: We are indebted to Francisco Queiroz for his expertise and help in the quantification of ATP levels.

Conflicts of Interest: D.A.S. is a consultant to, inventor of patents licensed to, and, in some cases, board member and investor of MetroBiotech, CohBar, Life Biosciences and affiliates, InsideTracker, Vium, Zymo, EdenRoc Sciences and affiliates, Immetas, Segterra, Galilei Biosciences, and Iduna Therapeutics. He is also an inventor on patent applications licensed to Bayer Crops, Merck KGaA, and Elysium Health. For details see https://genetics.med.harvard.edu/sinclair. The other authors report no conflicts of interest.

\section{References}

1. Martins, R.M.; Pinto Rolo, A.; Soeiro Teodoro, J.; Furtado, E.; Caetano Oliveira, R.; Tralhão, J.G.; Marques Palmeira, C. Addition of Berberine to Preservation Solution in an Animal Model of Ex Vivo Liver Transplant Preserves Mitochondrial Function and Bioenergetics from the Damage Induced by Ischemia/Reperfusion. Int. J. Mol. Sci. 2011, 19, 184-189. [CrossRef] [PubMed]

2. Martins, R.M.; Teodoro, J.S.; Furtado, E.; Rolo, A.P.; Palmeira, C.M.; Tralhão, J.G. Recent insights into mitochondrial targeting strategies in liver transplantation. Int. J. Med Sci. 2018, 15, 248. [CrossRef] [PubMed]

3. Jaeschke, H. Molecular mechanisms of hepatic ischemia-reperfusion injury and preconditioning. Am. J. Physiol. Gastrointest. Liver Physiol. 2003, 284, G15-G26. [CrossRef]

4. Carden, D.L.; Granger, D.N. Pathophysiology of ischaemia-reperfusion injury. J. Pathol. 2000, 190, $255-266$. [CrossRef]

5. Guan, L.-Y. Mechanisms of hepatic ischemia-reperfusion injury and protective effects of nitric oxide. World J. Gastrointest. Surg. 2014, 6, 122. [CrossRef] [PubMed]

6. Alexandrino, H.; Varela, A.T.; Teodoro, J.S.; Martins, M.A.; Rolo, A.P.; Tralhão, J.G.; Palmeira, C.M.; Castro e Sousa, F. Mitochondrial bioenergetics and posthepatectomy liver dysfunction. Eur. J. Clin. Investig. 2016, 46, 627-635. [CrossRef] [PubMed]

7. Varela, A.T.; Simões, A.M.; Teodoro, J.S.; Duarte, F.V.; Gomes, A.P.; Palmeira, C.M.; Rolo, A.P. Indirubin-3'-oxime prevents hepatic I/R damage by inhibiting GSK-3 [beta] and mitochondrial permeability transition. Mitochondrion 2010, 10, 456-463. [CrossRef]

8. Varela, A.T.; Rolo, A.P.; Palmeira, C.M. Fatty liver and ischemia/reperfusion: Are there drugs able to mitigate injury? Curr. Med. Chem. 2011, 18, 4987-5002. [CrossRef]

9. Camara, A.K.S.; Bienengraeber, M.; Stowe, D.F. Mitochondrial approaches to protect against cardiac ischemia and reperfusion injury. Front. Physiol. 2011, 2, 13. [CrossRef]

10. Jassem, W.; Heaton, N.D. The role of mitochondria in ischemia/reperfusion injury in organ transplantation. Kidney Int. 2004, 66, 514-517. [CrossRef]

11. Jassem, W.; Fuggle, S.V.; Rela, M.; Koo, D.D.; Heaton, N.D. The role of mitochondria in ischemia/reperfusion injury. Transplantation 2002, 73, 493-499. [CrossRef] [PubMed]

12. Katwal, G.; Baral, D.; Fan, X.; Weiyang, H.; Zhang, X.; Ling, L.; Xiong, Y.; Ye, Q.; Wang, Y. SIRT3 a major player in attenuation of hepatic ischemia-reperfusion injury by reducing ROS via its downstream mediators: SOD2, CYP-D, and HIF-1a. Oxidative Med. Cell. Longev. 2018, 2018, 2976957. [CrossRef] [PubMed]

13. Lejay, A.; Meyer, A.; Schlagowski, A.I.; Charles, A.L.; Singh, F.; Bouitbir, J.; Pottecher, J.; Chakfé, N.; Zoll, J.; Geny, B. Mitochondria: Mitochondrial participation in ischemia-reperfusion injury in skeletal muscle. Int. J. Biochem. Cell Biol. 2014, 50, 101-105. [CrossRef] [PubMed]

14. Palmeira, C.M.; Teodoro, J.S.; Amorim, J.A.; Steegborn, C.; Sinclair, D.A.; Rolo, A.P. Mitohormesis and metabolic health: The interplay between ROS, cAMP and sirtuins. Free Radic. Biol. Med. 2019, 141, $483-491$. [CrossRef]

15. Acin-Perez, R.; Salazar, E.; Kamenetsky, M.; Buck, J.; Levin, L.R.; Manfredi, G. Cyclic AMP Produced inside Mitochondria Regulates Oxidative Phosphorylation. Cell Metab. 2009, 9, 265-276. [CrossRef]

16. Di Benedetto, G.; Gerbino, A.; Lefkimmiatis, K. Shaping mitochondrial dynamics: The role of cAMP signaling. Biochem. Biophys. Res. Commun. 2018, 500, 65-74. [CrossRef]

17. Acin-Perez, R.; Russwurm, M.; Günnewig, K.; Gertz, M.; Zoidl, G.; Ramos, L.; Buck, J.; Levin, L.R.; Rassow, J.; Manfredi, G.; et al. A phosphodiesterase 2A isoform localized to mitochondria regulates respiration. J. Biol. Chem. 2011, 286, 30423-30432. [CrossRef] 
18. Zippin, J.H.; Chen, Y.; Nahirney, P.; Kamenetsky, M.; Wuttke, M.S.; Fischman, D.A.; Levin, L.R.; Buck, J. Compartmentalization of bicarbonate-sensitive adenylyl cyclase in distinct signaling microdomains. FASEB J. 2003, 17, 82-84. [CrossRef]

19. Wang, Z.; Liu, D.; Varin, A.; Nicolas, V.; Courilleau, D.; Mateo, P.; Caubère, C.; Rouet, P.; Gomez, A.M.; Vandecasteele, G.; et al. A cardiac mitochondrial cAMP signaling pathway regulates calcium accumulation, permeability transition and cell death. Cell Death Dis. 2016, 7, e2198. [CrossRef]

20. Signorile, A.; Santeramo, A.; Tamma, G.; Pellegrino, T.; D’Oria, S.; Lattanzio, P.; De Rasmo, D. Mitochondrial cAMP prevents apoptosis modulating Sirt3 protein level and OPA1 processing in cardiac myoblast cells. Biochim. Biophys. Acta Mol. Cell Res. 2017, 1864, 355-366. [CrossRef]

21. Scher, M.B.; Vaquero, A.; Reinberg, D. SirT3 is a nuclear NAD+-dependent histone deacetylase that translocates to the mitochondria upon cellular stress. Genes Dev. 2007, 21, 920-928. [CrossRef] [PubMed]

22. Singh, C.K.; Chhabra, G.; Ndiaye, M.A.; Garcia-Peterson, L.M.; MacK, N.J.; Ahmad, N. The Role of Sirtuins in Antioxidant and Redox Signaling. Antioxid. Redox Signal. 2018, 28, 643-661. [CrossRef] [PubMed]

23. Qiu, X.; Brown, K.; Hirschey, M.D.; Verdin, E.; Chen, D. Calorie restriction reduces oxidative stress by SIRT3-mediated SOD2 activation. Cell Metab. 2010, 12, 662-667. [CrossRef] [PubMed]

24. Someya, S.; Yu, W.; Hallows, W.C.; Xu, J.; Vann, J.M.; Leeuwenburgh, C.; Tanokura, M.; Denu, J.M.; Prolla, T.A. Sirt3 mediates reduction of oxidative damage and prevention of age-related hearing loss under Caloric Restriction. Cell 2010, 143, 802-812. [CrossRef] [PubMed]

25. Buler, M.; Aatsinki, S.M.; Izzi, V.; Hakkola, J. Metformin Reduces Hepatic Expression of SIRT3, the Mitochondrial Deacetylase Controlling Energy Metabolism. PLoS ONE 2012, 7, e49863. [CrossRef]

26. Anderson, K.A.; Hirschey, M.D. Mitochondrial protein acetylation regulates metabolism. Essays Biochem. 2012, 52, 23-35.

27. Carrico, C.; Meyer, J.G.; He, W.; Gibson, B.W.; Verdin, E. The Mitochondrial Acylome Emerges: Proteomics, Regulation by Sirtuins, and Metabolic and Disease Implications. Cell Metab. 2018, 27, 497-512. [CrossRef]

28. Kalogeris, T.; Baines, C.P.; Krenz, M.; Korthuis, R.J. Cell Biology of Ischemia/Reperfusion Injury. Int. Rev. Cell Mol. Biol. 2012, 298, 229-317.

29. Ristow, M.; Zarse, K. How increased oxidative stress promotes longevity and metabolic health: The concept of mitochondrial hormesis (mitohormesis). Exp. Gerontol. 2010, 45, 410-418. [CrossRef]

30. Lefkimmiatis, K.; Leronni, D.; Hofer, A.M. The inner and outer compartments of mitochondria are sites of distinct cAMP/PKA signaling dynamics. J. Cell Biol. 2013, 202, 453-462. [CrossRef]

31. Valsecchi, F.; Konrad, C.; Manfredi, G. Role of soluble adenylyl cyclase in mitochondria. Biochim. Biophys. Acta Mol. Basis Dis. 2014, 1842, 2555-2560. [CrossRef] [PubMed]

32. Di Benedetto, G.; Scalzotto, E.; Mongillo, M.; Pozzan, T. Mitochondrial $\mathrm{Ca}^{2+}$ uptake induces cyclic AMP generation in the matrix and modulates organelle ATP levels. Cell Metab. 2013, 17, 965-975. [CrossRef] [PubMed]

33. Valsecchi, F.; Konrad, C.; D'Aurelio, M.; Ramos-Espiritu, L.S.; Stepanova, A.; Burstein, S.R.; Galkin, A.; Magranè, J.; Starkov, A.; Buck, J.; et al. Distinct intracellular sAC-cAMP domains regulate ER Ca ${ }^{2+}$ signaling and OXPHOS function. J. Cell Sci. 2017, 130, 3713-3727. [CrossRef]

34. Ramos-Espiritu, L.; Kleinboelting, S.; Navarrete, F.A.; Alvau, A.; Visconti, P.E.; Valsecchi, F.; Starkov, A.; Manfredi, G.; Buck, H.; Adura, C.; et al. Discovery of LRE1 as a specific and allosteric inhibitor of soluble adenylyl cyclase. Nat. Chem. Biol. 2016, 12, 838-844. [CrossRef]

35. Puigserver, P.; Spiegelman, B.M. Peroxisome proliferator-activated receptor-gamma coactivator 1 alpha (PGC-1 alpha): Transcriptional coactivator and metabolic regulator. Endocr. Rev. 2003, 24, 78-90. [CrossRef] [PubMed]

36. Youle, R.J.; Narendra, D.P. Mechanisms of mitophagy. Nat. Rev. Mol. Cell Biol. 2011, 12, 9-14. [CrossRef] [PubMed]

37. Isobe, K.; Jung, H.J.; Yang, C.R.; Claxton, J.N.; Sandoval, P.; Burg, M.B.; Raghuram, V.; Knepper, M.A. Systems-level identification of PKA-dependent signaling in epithelial cells. Proc. Natl. Acad. Sci. USA 2017, 114, E8875-E8884. [CrossRef]

38. Turnham, R.E.; Scott, J.D. Protein kinase A catalytic subunit isoform PRKACA; History, function and physiology. Gene 2016, 577, 101-108. [CrossRef]

39. Sun, W.; Liu, C.; Chen, Q.; Liu, N.; Yan, Y.; Liu, B. SIRT3: A new regulator of cardiovascular diseases. Oxidative Med. Cell. Longev. 2018, 2018, 7293861. [CrossRef] 
40. Rossetti, A.; Togliatto, G.; Rolo, A.P.; Teodoro, J.S.; Granata, R.; Ghigo, E.; Columbano, A.; Palmeira, C.M.; Brizzi, M.F. Unacylated ghrelin prevents mitochondrial dysfunction in a model of ischemia/reperfusion liver injury. Cell Death Discov. 2017, 3, 1-11. [CrossRef]

41. Teodoro, J.S.; Varela, A.T.; Duarte, F.V.; Gomes, A.P.; Palmeira, C.M.; Rolo, A.P. Indirubin and NAD ${ }^{+}$prevent mitochondrial ischaemia/reperfusion damage in fatty livers. Eur. J. Clin. Investig. 2018, 48, e12932. [CrossRef] [PubMed]

42. Teodoro, J.S.; Palmeira, C.M.; Rolo, A.P. Mitochondrial membrane potential $(\Delta \Psi)$ fluctuations associated with the metabolic states of mitochondria. Methods Mol. Biol. 2018, 1782, 109-119.

43. Palmeira, C.M.; Moreno, A.J.; Madeira, V.M.C. Interactions of herbicides 2,4-D and dinoseb with liver mitochondrial bioenergetics. Toxicol. Appl. Pharmacol. 1994, 127, 50-57. [CrossRef] [PubMed]

44. Gornall, A.G.; Bardawill, C.J.; David, M.M. Determination of serum proteins by means of the biuret reaction. J. Biol. Chem. 1949, 177, 751-766. [PubMed]

45. Rolo, A.P.; Oliveira, P.J.; Moreno, A.J.; Palmeira, C.M. Bile acids affect liver mitochondrial bioenergetics: Possible relevance for cholestasis therapy. Toxicol. Sci. Off. J. Soc. Toxicol. 2000, 57, 177-185. [CrossRef] [PubMed]

46. Chance, B.; Williams, G.R. The respiratory chain and oxidative phosphorylation. Adv. Enzymol. Relat. Subj. Biochem. 2006, 17, 65-134.

47. Kamo, N.; Muratsugu, M.; Hongoh, R.; Kobatake, Y. Membrane potential of mitochondria measured with an electrode sensitive to tetraphenyl phosphonium and relationship between proton electrochemical potential and phosphorylation potential in steady state. J. Membr. Biol. 1979, 49, 105-121. [CrossRef]

48. Palmeira, C.M.; Wallace, K.B. Benzoquinone inhibits the voltage-dependent induction of the mitochondrial permeability transition caused by redox-cycling naphthoquinones. Toxicol. Appl. Pharmacol. 1997, 143, 338-347. [CrossRef]

49. Zhou, S.; Palmeira, C.M.; Wallace, K.B. Doxorubicin-induced persistent oxidative stress to cardiac myocytes. Toxicol. Lett. 2001, 121, 151-157. [CrossRef]

50. Rio, D.C.; Ares, M.; Hannon, G.J.; Nilsen, T.W. Purification of RNA using TRIzol (TRI Reagent). Cold Spring Harb. Protoc. 2010, 5, pdb-prot5439. [CrossRef]

51. Livak, K.J.; Schmittgen, T.D. Analysis of relative gene expression data using real-time quantitative PCR and the 2- $\Delta \Delta C$ T method. Methods 2001, 25, 402-408. [CrossRef] [PubMed] 